\title{
Efectos del clima y capacidad de almacenamiento de agua del suelo en la productividad de rodales de pino radiata en Chile: un análisis utilizando el modelo 3-PG
}

\author{
Climatic and soil water storage capacity effects on radiata pine \\ productivity in Chile: An analysis using the model 3-PG
}

\author{
FRANCISCO J. FLORES, H. LEE ALLEN
}

North Carolina State University, 3108 Jordan Hall, Box 8008 Raleigh, NC 27695, USA

E-mail: fjflores@unity.ncsu.edu, lallen@ncsfnc.cfr.ncsu.edu

\section{SUMMARY}

\begin{abstract}
Radiata pine (Pinus radiata, D. Don) is planted across a wide range of soils and climate in Chile, and plantations exhibit a great variation in productivity. In order to effectively manage, we need to quantify potential productivity, determine the principal non-manipulated site and stand factors that determine the potential, identify which stands are currently performing below their expectations, determine which soil and/or stand limitations result in suboptimal production in these stands, and develop silvicultural treatment regimes to effectively ameliorate the identified limitations. To estimate potential productivity, we used the ecophysiological model 3-PG to provide estimates of stem wood production for fully stocked radiata pine plantations located in the VI to IX regions of Chile. 3-PG was also used to explore the effects of climate and soil water storage capacity on potential productivity. Potential productivity estimates for radiata pine from 3-PG ranged from 14 to $44 \mathrm{~m}^{3} \mathrm{ha}^{-1} \mathrm{yr}-{ }^{1}$. Within the study area, precipitation was responsible for much of the variation in potential productivity. The model indicated considerable variation in the extent and magnitude of soil water limitations.
\end{abstract}

Key words: Pinus radiata, 3-PG, potential productivity, process models, Chile.

\section{RESUMEN}

En Chile, pino radiata (Pinus radiata D. Don) ha sido plantado a lo largo de un amplio rango de suelos y climas, y las plantaciones exhiben amplias variaciones en productividad. Para manejar eficientemente la productividad, es necesario cuantificar la productividad potencial, identificar los principales factores no-manipulables que determinan ese potencial, identificar qué rodales tienen una producción bajo el potencial, determinar qué limitaciones de suelo y/o del rodal son responsables de los niveles de producción del rodal bajo el óptimo, y desarrollar tratamientos silviculturales que mejoran efectivamente las limitaciones que han sido identificadas. Para estimar la productividad potencial, fue utilizado el modelo ecofisiológico 3-PG, generando estimaciones de producción de madera del fuste de rodales de pino radiata de densidad normal, ubicados entre la VI y IX Regiones de Chile. 3-PG, también fue utilizado para explorar los efectos del clima y capacidad de almacenamiento de agua del suelo sobre la productividad potencial. Las estimaciones de productividad potencial de pino radiata con 3-PG variaron entre 14 y $44 \mathrm{~m}^{3} \mathrm{ha}^{-1} \mathrm{año}^{-1}$. Dentro del área de estudio, la mayor parte de la variación en la productividad potencial se debió a la precipitación. El modelo indicó variaciones considerables en la extensión y magnitud de las limitaciones de agua del suelo.

Palabras clave: Pinus radiata, 3-PG, productividad potencial, modelos de proceso, Chile. 
BOSQUE 25(3): 11-24, 2004

Efectos del clima y capacidad de almacenamiento de agua del suelo en productividad de rodales de pino radiata en Chile...

\section{INTRODUCCION}

La productividad de una plantación forestal es determinada básicamente por el área foliar y las relaciones suelo-planta-atmósfera. Estas últimas incluyen, por ejemplo, el efecto que tiene el déficit de presión de vapor o la cantidad de agua del suelo sobre las tasas de fotosíntesis de una plantación. El área foliar, medida a través del índice de área foliar (IAF), determina la cantidad de radiación solar fotosintéticamente activa absorbida, la que se encuentra estrechamente relacionada con la productividad primaria neta (PPN) de una plantación (1-4).

Para lograr optimizar el manejo de plantaciones de pino radiata (Pinus radiata D. Don) es necesario conocer el efecto de las condiciones meteorológicas y su interacción con factores de suelo (por ejemplo: capacidad de almacenamiento de agua) en la producción de madera. Si bien no es posible manejar las condiciones meteorológicas, es posible determinar climas más favorables para el crecimiento de pino radiata, permitiendo estimar rangos de productividad potencial de esta especie en distintos sitios.

Para predecir el crecimiento y desarrollo de rodales bajo distintas condiciones de sitio y manejo se utilizan modelos de crecimiento. Estos modelos pueden ser clasificados en dos tipos generales: 1) Modelos de crecimiento y rendimiento y 2) Modelos de procesos. Los modelos de crecimiento y rendimiento son representaciones estadísticas del desarrollo de un rodal a través del tiempo. Para desarrollar estos modelos es necesario medir las variables de interés (número de árboles por hectárea, área basal, volumen o altura), a través del tiempo y en distintas condiciones de sitio y manejo. Puesto que las combinaciones posibles de sitio y manejo son muy altas, generalmente los datos utilizados para ajustar los modelos cubren sólo un rango limitado de condiciones de crecimiento. Adicionalmente, los modelos de crecimiento y rendimiento son una representación del crecimiento y desarrollo de rodales bajo las condiciones de manejo utilizadas en el pasado, en las cuales se consideran las condiciones del sitio como fijas y no necesariamente reflejan el crecimiento potencial bajo un esquema de manejo intensivo.

Los modelos de procesos, por otra parte, utilizan funciones basadas en datos experimentales o la mejor representación teórica de los procesos fisiológicos que determinan el crecimiento de los árboles. En este caso, el crecimiento de un rodal no se modela directamente como función del tiempo, sino que es el resultado de la interacción de distintos procesos fisiológicos con las condiciones meteorológicas y de sitio en un período de tiempo determinado. La idea es incluir en forma explicita, relaciones causa-efecto entre variables meteorológicas y de suelo y los procesos fisiológicos que finalmente regulan el crecimiento de los árboles. Para ajustar estos modelos, es necesario medir la respuesta de los procesos fisiológicos en relación con las condiciones meteorológicas y de suelo. Estos modelos, en su estado de desarrollo actual, son utilizados principalmente como herramientas de investigación destinadas a entender y sintetizar el conocimiento actual acerca de los factores que determinan el crecimiento, junto con identificar áreas que requieren más investigación (5). Entre las salidas (output) más comunes en este tipo de modelos se incluye: Productividad primaria bruta y neta, evapotranspiración, fluctuaciones en el contenido de humedad del suelo y conductancia del dosel. Las principales limitaciones de este tipo de modelos incluyen una representación limitada de la estructura del rodal, la falta de conexión con módulos de análisis económico y la falta de un conocimiento completo de los procesos fisiológicos que determinan la producción de madera $(6,7)$. Si bien es difícil, al menos en su estado actual, utilizar estos modelos para proyectar el crecimiento y desarrollo de un rodal, existen grandes oportunidades para utilizarlos como parte de un sistema de actualización de inventarios.

El amplio rango de distribución de pino radiata en Chile, entre los paralelos $30^{\circ}$ y $41^{\circ}$ de latitud sur, incluye una gran variedad de condiciones climáticas, destacando especialmente el amplio rango de precipitaciones, desde los 500 a más de $3.200 \mathrm{~mm}$ por año. Estas amplias variaciones climáticas provocan variaciones importantes en la productividad de pino radiata. Estas variaciones han sido documentadas en el trabajo de Gerding y Schlatter (8) que analizó diferencias en índice de sitio asociadas a distintos factores del clima y suelo (propiedades físicas y químicas). Ellos concluyeron que el régimen hídrico era el principal factor que explicaba las diferencias en productividad. Sin embargo, al utilizar datos empíricos a lo largo de 
zonas con amplias variaciones de suelo y clima, no es tan claro poder separar el efecto de la fertilidad del suelo, clima o manejo de las plantaciones y, por lo tanto, es necesario realizar nuevos estudios para dilucidar estas interrogantes.

Gerding y Schlatter (8) mostraron también que la productividad varía ampliamente dentro de cada zona climática y tipo de suelo. Sus resultados sugieren que debiera haber una alta probabilidad de incrementar la productividad, desde el punto de vista biológico y económico, en sitios que presentan una productividad inferior al máximo observado para la unidad de suelo-clima en que se encuentran. Las respuestas positivas en crecimiento que han sido observadas luego del control de la vegetación competidora $(9,10)$ y/o fertilización (11) indican también que los niveles actuales de producción parecen estar muy por debajo de los niveles potenciales en muchas plantaciones de pino radiata. La productividad de una plantación es una función de factores de sitio y del rodal tanto no manipulables (radiación, precipitación, textura del suelo) como manipulables (profundidad de enraizamiento, fertilidad del suelo, vegetación competidora, densidad del rodal). Para lograr manejar la productividad en forma efectiva es necesario cuantificar la productividad potencial, determinar el principal factor no manipulable que determina ese potencial, identificar qué rodales están produciendo bajo ese potencial, determinar limitaciones de suelo y/o del rodal que resultan en los niveles de producción por debajo del óptimo en esos rodales, y desarrollar regímenes de tratamientos silviculturales que mejoren en forma efectiva las limitaciones que han sido identificadas $(4,12)$.

El presente estudio tiene como objetivo entender mejor cómo los factores de suelo y clima interactúan determinando el período de déficit hídrico y la productividad de las plantaciones de pino radiata en Chile. Esto permitirá comparar, al menos en forma relativa, el crecimiento anual en volumen en distintas zonas climáticas del país y determinar los factores climáticos más importantes que controlan la productividad potencial de pino radiata en Chile y su interacción con la capacidad de almacenamiento de agua del suelo.

\section{MATERIAL Y METODOS}

Los datos climáticos utilizados en las simulaciones fueron obtenidos del Atlas Agroclimático de Chile para las Regiones VI, VII, VIII y IX, con mapas a escala 1: 500.000 (13). Este atlas incluye diferentes zonas climáticas determinadas por los siguientes parámetros: Régimen de heladas, régimen térmico global, estacionalidad térmica (invierno y verano), régimen hídrico global y estacionalidad hídrica (invierno y verano). Para cada una de las zonas climáticas definidas existe información media mensual de temperatura máxima y mínima, precipitación, radiación solar y número de heladas. Como los datos climáticos están asociados a zonas climáticas que en su mayoría abarcan grandes extensiones de terreno, la resolución espacial del presente análisis es limitada. Esta limitación es especialmente importante en el sentido norte sur.

En el presente análisis, fueron incluidas las unidades climáticas desde el paralelo $35^{\circ}$ latitud Sur, hasta el límite sur de la IX Región, ubicado alrededor del paralelo $39^{\circ}$ latitud Sur (figura 1). Además, fueron excluidas las zonas altas de la Cordillera de Nahuelbuta y medias a altas de la Cordillera de Los Andes, por la nula o escasa presencia de plantaciones de pino radiata en esas zonas.

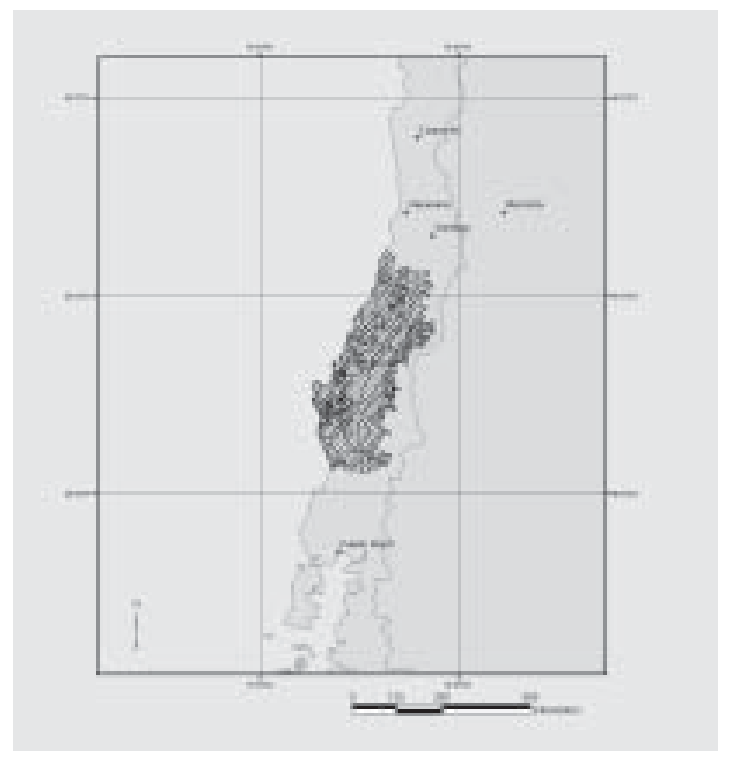

Figura 1. Mapa de ubicación indicando la zona estudiada.

Location map of the study area. 
BOSQUE 25(3): 11-24, 2004

Efectos del clima y capacidad de almacenamiento de agua del suelo en productividad de rodales de pino radiata en Chile...

La productividad primaria neta fue calculada utilizando el modelo de procesos 3-PG (14), asumiendo un índice de área foliar de $4 \mathrm{~m}^{2} \mathrm{~m}^{-2}$, una textura franco arcillosa y variando la capacidad de almacenamiento de agua del suelo entre $50 \mathrm{y}$ $500 \mathrm{~mm}$.

Las salidas de estas simulaciones fueron utilizadas para realizar un análisis de sensibilidad del crecimiento de pino radiata respecto de variables de suelo (capacidad de almacenamiento de agua) y climáticas (precipitación, déficit de presión de vapor y radiación solar).

El modelo 3-PG ha sido utilizado en diversas zonas geográficas, como Australia, Nueva Zelanda, Brasil, Sureste y Noroeste de Estados Unidos, Suecia y el Reino Unido, y aplicado a una gran variedad de especies, como Pinus radiata, Eucalyptus globulus, Eucalyptus nitens, Pinus taeda, Picea abies, Picea sitchensis y Pseudotsuga menziesii, entre otros $(7,15-20)$.

3-PG (figura 2) es un modelo de procesos simple, que utiliza datos climáticos mensuales, inclu- yendo variables fácilmente disponibles como temperatura máxima y mínima promedio, precipitación y radiación solar. Como salidas del modelo se incluye biomasa del fuste, hojas y raíces, atributos de rodal convencionales como volumen, área basal y número de árboles por hectárea, y parámetros de interés ecofisiológico como índice de área foliar, conductancia del dosel, tasa de transpiración y contenido de agua en el suelo. 3-PG cuenta con cinco componentes (módulos) principales:

- Producción de Biomasa: Basado en el concepto de "las hojas hacen que los árboles crezcan", es decir, a mayor área foliar, mayor absorción de radiación solar y, por tanto, mayor producción de biomasa. La eficiencia con que esa radiación absorbida es convertida a biomasa cambia dependiendo de las condiciones del medioambiente. Por otra parte, el modelo asume una fracción fija de respiración $(53 \%)$ para calcular la producción neta del dosel.

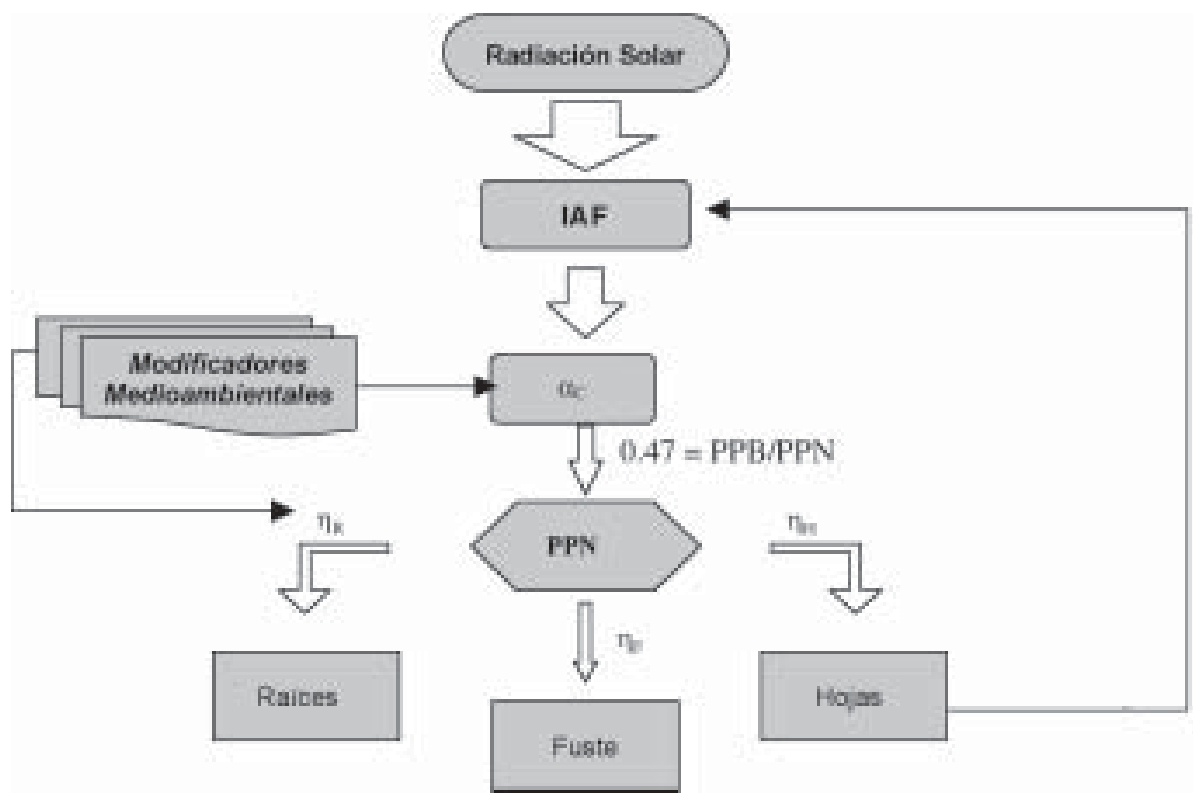

Figura 2. Estructura fundamental de 3-PG. IAF es el índice de área foliar; $\alpha_{C}$ es la eficiencia en el uso de la luz; $\eta_{\mathrm{R}}, \eta_{\mathrm{F}}$ y $\eta_{\mathrm{H}}$ son coeficientes de partición de la biomasa para raíces, fuste y hojas, respectivamente; PPB y PPN son productividad primaria bruta y neta respectivamente (adaptado de Landsberg and Waring (14)).

Fundamental structure of 3-PG. IAF is leaf area index; $\alpha_{C}$ is light use efficiency; $\eta_{R}, \eta_{F}$ y $\eta_{H}$ are biomass partitioning coefficients for roots, stem and foliage, respectively; PPB and PPN are gross and net primary productivity, respectively (adapted from Landsberg and Waring (14)). 
- Partición de la Biomasa: La biomasa producida es distribuida en tres componentes: fuste, hojas y raíces. La proporción de biomasa producida cada mes que se asigna a cada componente depende de las condiciones de crecimiento y tamaño de los árboles.

- Mortalidad: Es calculada basándose en la ley de autorraleo.

- Balance de Agua en el Suelo: Para calcular el balance hídrico se utiliza el volumen de suelo de la zona de arraigamiento, la cual es caracterizada en términos de su capacidad de almacenamiento de agua disponible y textura (asumiendo homogeneidad en estas propiedades). La pérdida por evapotranspiración es determinada con la ecuación Penman-Monteith. Además, se asume una pérdida fija por intercepción del dosel.

- Atributos del Rodal: Son calculados a partir de la cantidad de biomasa de los distintos componentes, supuestos acerca de la fracción de corteza y ramas, densidad de la madera y relaciones alométricas.

Para calcular la cantidad de biomasa producida, 3-PG ajusta la eficiencia en el uso de la luz, por limitaciones impuestas por el medioambiente (14). Los modificadores (cuadro 1) reflejan limitaciones en la utilización de la radiación solar por parte del follaje, impuestas por el cierre de los estomas, producto de la falta de agua en el suelo o por alto déficit de presión de vapor, por efecto de la temperatura y heladas.

\section{CUADRO}

Modificadores medioambientales en 3-PG (Landsberg and Waring (14)).

Environmental modifiers in 3-PG (Landsberg and Waring (14))

\begin{tabular}{|ll|}
\hline Factores & Modificadores \\
\hline Déficit de presión de vapor & $f_{D P V}(D)$ \\
Agua del suelo & $f_{A S}(\theta)$ \\
Temperatura & $f_{T}\left(T_{\text {media }}\right)$ \\
Heladas & $F_{H}\left(d_{h}\right)$ \\
Fertilidad & $f_{F}(F R)$ \\
\hline
\end{tabular}

De esta forma, la eficiencia máxima es reducida a través de modificadores que toman valores entre 0 y 1 , como se observa en la siguiente ecuación:

$$
\alpha_{C}=f_{T} f_{H} f_{F} \min \left\{f_{D P V} f_{A S}\right\} \alpha_{C x}
$$

Donde:

$\alpha_{\mathrm{C}}$ : Eficiencia en el uso de la luz $\left(\mathrm{gMJ}^{-2}\right)$

$\alpha_{\mathrm{Cx}}$ : Eficiencia máxima en el uso de la luz $\left(\mathrm{gMJ}^{-2}\right)$

$f_{T}, f_{H}, f_{F}, f_{D P V}, f_{A S}:$ Modificadores medioambientales especificados en el cuadro 1 .

La producción bruta del dosel es calculada utilizando la ley de Beer para determinar la cantidad de radiación solar que es interceptada por el dosel, y ese valor es multiplicado por la eficiencia en el uso de la luz que ha sido ajustada a través de los modificadores medioambientales. La siguiente ecuación muestra la forma de cálculo de la producción bruta del dosel:

$$
\mathrm{P}_{\mathrm{b}}=\alpha_{\mathrm{C}}\left(1-e^{-k L}\right) \mathrm{Q}_{0}
$$

donde:

$\mathrm{P}_{\mathrm{b}}$ : Producción bruta del dosel (ton $\mathrm{ha}^{-1} \mathrm{a}^{-1}$ )

$\alpha_{C}$ : Eficiencia en el uso de la luz, ajustada por modificadores medioambientales $\left(\mathrm{gMJ}^{-2}\right)$

$k$ : $\quad$ Coeficiente de extinción de la luz

$L: \quad$ índice de área foliar $\left(\mathrm{m}^{2} \mathrm{~m}^{-2}\right)$

$\mathrm{Q}_{0}$ : radiación solar fotosintéticamente activa que es recibida en el sitio $\left(\mathrm{MJm}^{-2} \mathrm{dí}^{-1}\right)$

Finalmente, la productividad primaria neta (PPN) es calculada multiplicando la producción bruta del dosel por 0,47 para descontar la respiración autotrófica (21).

Los mecanismos que determinan la distribución del carbono fijado a los distintos componentes del árbol, aún no han sido completamente dilucidados (14). El problema radica en la falta de información acerca de los factores que determinan la dinámica en el tiempo de la proporción de carbono distribuida al fuste, hojas o raíces. Teniendo en cuenta estas limitaciones, el modelo fue utilizado para calcular la productividad primaria neta (PPN), evitando de este modo distorsionar la comparación entre sitios producto de cambios en la distribución del carbono que es fijado cada año. 
Una vez calculados los valores de productividad primaria neta con 3-PG, estos fueron convertidos a incremento anual corriente en volumen utilizando la siguiente fórmula:

$$
\mathrm{IAC}=\mathrm{PPN} \times \eta_{\mathrm{F}} / \rho
$$

donde:

IAC: Incremento anual corriente en volumen. PPN: Productividad primaria neta (ton $\mathrm{ha}^{-1} \mathrm{a}^{-1}$ ).

$\eta_{\mathrm{F}}$ : Es la fracción de la productividad primaria neta que es distribuida al fuste. En este caso se asumió un valor de 0,4.

$\rho: \quad$ Densidad de la madera. Se asumió un valor de $400 \mathrm{~kg} \mathrm{~m}^{-3}$.

Los valores de incremento anual corriente calculados de esta forma deben ser considerados como una buena medida relativa de productividad. Variaciones regionales en densidad de la madera y distribución de la biomasa al fuste podrían causar discrepancias entre los valores calculados en este análisis y lo que es posible observar en terreno.

Parametrización del Modelo: En el presente análisis se utilizó 3-PG para estimar la productividad primaria neta (PPN), asumiendo un índice de área foliar (IAF) de $4 \mathrm{~m}^{2} \mathrm{~m}^{-2}$, representando una fertilidad alta y, por esto, estimando la productividad potencial de pino radiata. Si bien es cierto que las plantaciones de pino radiata en Chile se extienden en distintos tipos de suelo, en este trabajo se realizaron las simulaciones sólo para un suelo franco arcilloso. En el futuro es posible ampliar este estudio incluyendo el efecto de distintas texturas de suelo. A partir de estas condiciones de rodal y sitio base, se realizaron simulaciones con distintas capacidades de almacenamiento de agua disponible del suelo $(50$ a $500 \mathrm{~mm}$ ). De esta forma fue posible analizar el efecto del clima y su interacción con la capacidad de almacenamiento de agua del suelo sobre la PPN.

El índice de área foliar presenta una dinámica estacional con un máximo durante el verano y de acuerdo al estudio realizado por Whitehead et al. (22) en Nueva Zelanda, cambios de hasta un $100 \%$ desde invierno a verano son posibles. En estos momentos en Chile no existe información acerca de los patrones de despliegue del área foliar, retención de acículas y patrones de caída de la ho- jarasca, todos necesarios para determinar el patrón estacional del IAF. Por esta razón fue necesario asumir un valor fijo de área foliar para todo el año.

Para lograr un índice de área foliar de $4 \mathrm{~m}^{2} \mathrm{~m}^{-2}$ constante durante todo el año, fue necesario cambiar a 0 los parámetros que determinan la tasa de caída de las hojas y porcentaje de carbono fijado que es distribuido a hojas (sin pérdidas ni aumento hojas). Finalmente, se asignó un valor 0 a los parámetros que determinan el porcentaje de carbono fijado que es distribuido a raíces y, de esta forma, la variable de salida producción del fuste en 3-PG es en realidad el valor de PPN. Los otros parámetros del modelo, incluyendo los modificadores ambientales, fueron obtenidos a partir de Landsberg y Waring (14). Las corridas del modelo fueron realizadas en una planilla de cálculo y los resultados fueron asociados al mapa de distritos agroclimático de Chile (13) en el sistema de información geográfico ArcView.

\section{RESULTADOS Y DISCUSION}

El amplio rango de distribución de pino radiata en Chile, entre los paralelos $30^{\circ}$ y $41^{\circ}$ de latitud sur, incluye una gran variedad de condiciones climáticas, destacando especialmente el amplio rango de precipitaciones, desde los 500 a más de $3.200 \mathrm{~mm}$ por año. En este estudio se incluyó sólo la parte de la distribución de pino radiata comprendida entre los $35^{\circ}$ y $39^{\circ}$ de latitud sur. En esta parte del rango de distribución de la especie en Chile, las precipitaciones varían entre 600 y $3.200 \mathrm{~mm}$, aproximadamente. El efecto del anticiclón del Pacífico Suroriental determina que el clima sea mediterráneo, con lluvias concentradas en invierno y un período seco durante el verano que puede variar entre 1 y 7 meses. Otra característica importante es una correlación negativa entre la precipitación y la cantidad de radiación solar. Como se puede apreciar en la figura 3, no existen sitios con alta radiación solar y alta precipitación, o baja radiación solar y baja precipitación.

Al igual que con la radiación solar, existe una correlación negativa entre la precipitación y la temperatura media anual. La alta correlación entre precipitación y la radiación solar o temperatura media anual dificulta el uso del análisis esta- 


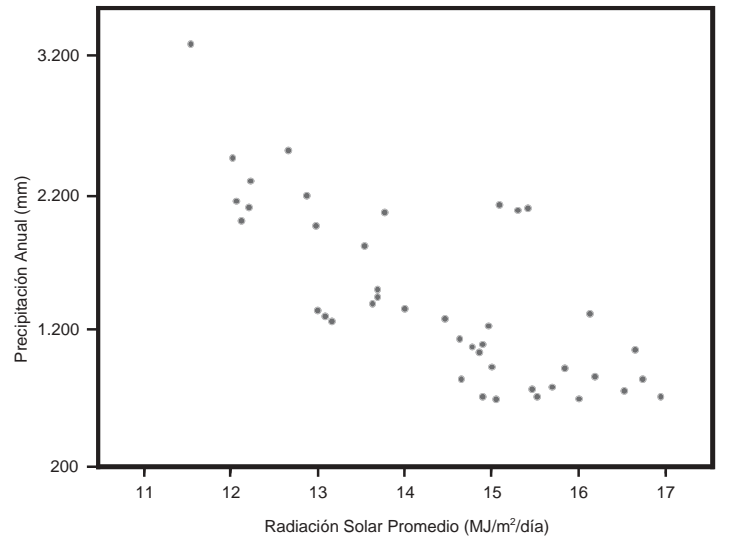

Figura 3. Dominio de precipitación anual versus radiación solar promedio anual para el área de distribución de pino radiata.

Annual-precipitation-solar-radiation domain for the distribution area of radiata pine.

dístico basado en regresiones entre productividad y estas variables climáticas, puesto que es difícil separar el efecto de una u otra. En este sentido, una de las ventajas de los modelos de procesos como 3-PG es que permiten un análisis explícito de las variables climáticas en términos de causa efecto.

Otra de las variables meteorológicas importantes para el crecimiento de los árboles es el déficit de presión de vapor, que mide el poder secante del aire. Si el déficit de presión de vapor es alto, la tasa de fotosíntesis neta disminuye producto del cierre parcial o total de los estomas que se traduce en una baja conductividad estomática (23). La figura 4, muestra la relación que existe entre el déficit de presión de vapor en septiembre y la precipitación anual. Se puede observar que, en general, existe una tendencia a disminución de la precipitación a medida que aumenta el déficit de presión de vapor. Sin embargo, a diferencia de lo que ocurre con la radiación solar, existen zonas con baja precipitación y bajo déficit de presión de vapor (zona costera de las Regiones VII y VIII).

Efectos del clima sobre la productividad de pino radiata: La gran variación que existe en las condiciones climáticas a lo largo del rango de distribución de pino radiata en Chile tiene un impacto importante en la productividad de las plantaciones. El incremento anual corriente en volumen,

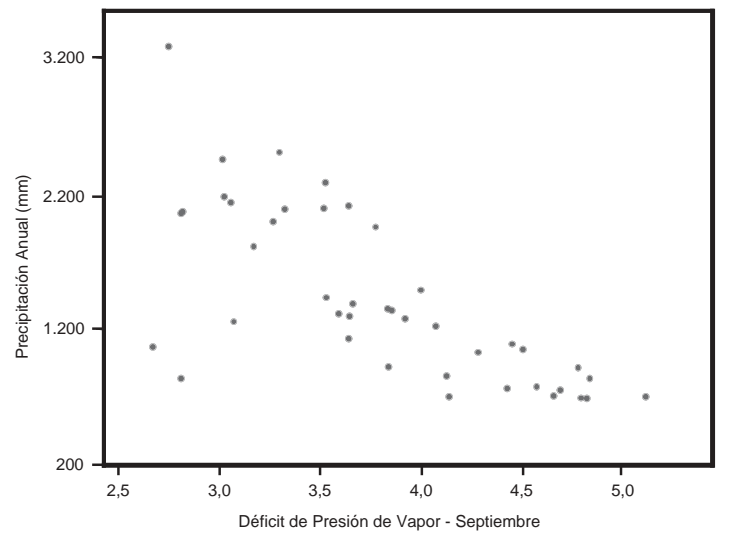

Figura 4. Dominio de precipitación versus déficit de presión de vapor para el área de distribución de pino radiata.

Precipitation-vapor pressure-deficit domain for the distribution area of radiata pine.

estimado en este estudio para un índice de área foliar de $4 \mathrm{~m}^{2} \mathrm{~m}^{-2}$, varía entre $14 \mathrm{~m}^{3} \mathrm{ha}^{-1}$ año $^{-1}$ y 44 $\mathrm{m}^{3} \mathrm{ha}^{-1}$ año $^{-1}$, para suelos con capacidad de almacenamiento de $50 \mathrm{~mm}$ y $500 \mathrm{~mm}$. El valor máximo encontrado, es levemente inferior al valor de incremento anual corriente máximo $\left(52 \mathrm{~m}^{3} \mathrm{ha}^{-1}\right.$ año ${ }^{-1}$ ) para un sitio clase 1 (índice de sitio 32) sin manejo en la zona de crecimiento 6 (Concepción, Arauco) reportado por Peters et al. (24) en base al simulador RADIATA. Una de las razones que pueden explicar esta diferencia incluyen la posibilidad de que el índice de área foliar sea mayor a $4 \mathrm{~m}^{2} \mathrm{~m}^{-2}$, como ha sido reportado en pino radiata en Australia (25). El otro factor que puede incidir en la subestimación es que el valor de materia seca que se particiona al fuste sea mayor a un $40 \%$, pudiendo alcanzar el 50\% (26).

La variación mensual en los modificadores medioambientales de 3-PG permite visualizar qué factores son los más restrictivos para el crecimiento de pino radiata a lo largo de las distintas zonas climáticas. Las figuras 5 y 6 , muestran la variación mensual de los cuatro modificadores medioambientales para dos zonas, la costa de Arauco y el Valle Central a la altura de Chillán, que reciben 1.260 y $1.093 \mathrm{~mm}$ de precipitación, respectivamente. A pesar de la escasa diferencia en precipitación, existen dramáticas diferencias en productividad, en el caso de Arauco el incremento anual corriente es de $38 \mathrm{~m}^{3} \mathrm{ha}^{-1} \mathrm{año}^{-1}$, y en Chillán es de $25 \mathrm{~m}^{3} \mathrm{ha}^{-1} \mathrm{año}^{-1}$. 


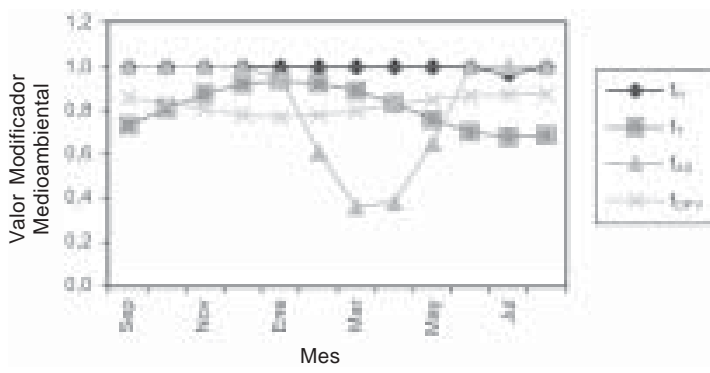

Figura 5. Modificadores ambientales de 3-PG para el área de Arauco, para suelos con $400 \mathrm{~mm}$ de capacidad de almacenamiento de agua.

3-PG environmental modifiers for Arauco. Soils with 400 $\mathrm{mm}$ of available water storage capacity.

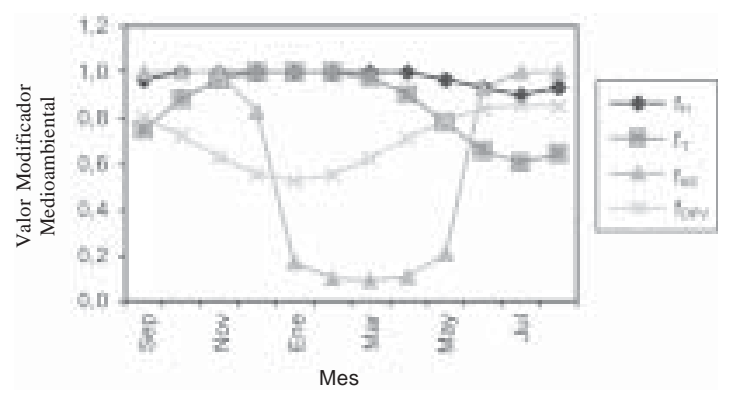

Figura 6. Modificadores ambientales de 3-PG para el área de Chillán, para suelos con $400 \mathrm{~mm}$ de capacidad de almacenamiento de agua.

3-PG environmental modifiers for Chillán. Soils with 400 $\mathrm{mm}$ of available water storage capacity.

En el caso de la costa de Arauco, se puede observar que la falta de agua en el suelo es el factor más limitante entre febrero y mayo. En el período septiembre-enero las limitaciones medioambientales son bastante moderadas, dominadas por temperatura en los primeros meses y por déficit de presión de vapor a comienzos del verano. Las limitaciones por temperatura vuelven a dominar durante los meses de invierno, y prácticamente no existen problemas de heladas.

En el caso del área de Chillán, las limitaciones por falta de agua del suelo son no sólo mayores en magnitud, sino también se extienden por un período mucho mayor (enero-mayo). En el período septiembre-diciembre existen limitaciones importantes producto del alto déficit de presión de vapor. En este período, como el poder secante del aire es alto, los estomas permanecen cerrados o parcialmente cerrados por mayor tiempo y con eso se reduce la eficiencia fotosintética. Por otra parte, las limitaciones por temperatura y heladas son más pronunciadas que en el caso anterior.

Las diferencias en la magnitud y duración del período de estrés hídrico, entre una zona y otra, pueden ser explicadas principalmente en términos de la mayor demanda evaporativa del aire en el caso de Chillán, puesto que la precipitación varía sólo en $150 \mathrm{~mm}$.

Análisis de sensibilidad de la productividad a variables climáticas: A continuación se presenta un análisis de sensibilidad del crecimiento anual corriente de pino radiata a distintas variables climáticas. Los gráficos presentados en esta sección incluyen una línea que muestra la tendencia de la relación entre las variables presentadas. No se realizaron análisis estadísticos para determinar la mejor línea de regresión puesto que los datos utilizados no son empíricos, sino simulados en forma determinística. La figura 7 muestra cómo el crecimiento de pino radiata disminuye a medida que aumenta la radiación solar. Esto no es producto de un efecto directo de la radiación solar sobre el crecimiento de pino radiata, sino más bien producto de la correlación con otros factores climáticos, como ocurre con la precipitación (situación particular del clima en Chile).

En el caso de la precipitación anual (figura 8) es posible observar un claro incremento en el crecimiento de pino radiata, a medida que aumentan las precipitaciones. El aumento en la productividad es prácticamente lineal hasta aproximadamente $1.500 \mathrm{~mm}$. Estos resultados concuerdan con lo encontrado por Gerding y Schlatter (8) utilizando datos empíricos de productividad. Nuestro análisis está basado en estimaciones de productividad en condiciones de alta fertilidad y de este modo permite analizar en forma clara el efecto de las condiciones climáticas y capacidad de almacenamiento de agua (separando el efecto de la nutrición).

En el caso de suelos con $400 \mathrm{~mm}$ de capacidad de almacenamiento de agua, existen zonas de alta productividad, incluso con precipitaciones menores a $1.000 \mathrm{~mm}$ (dentro de elipse en figura 8). Estos sitios de alta eficiencia productiva están ubicados a lo largo de la costa desde las Regiones VII a la IX.

Existe una relación negativa entre el crecimiento de pino radiata y el déficit de presión de vapor 


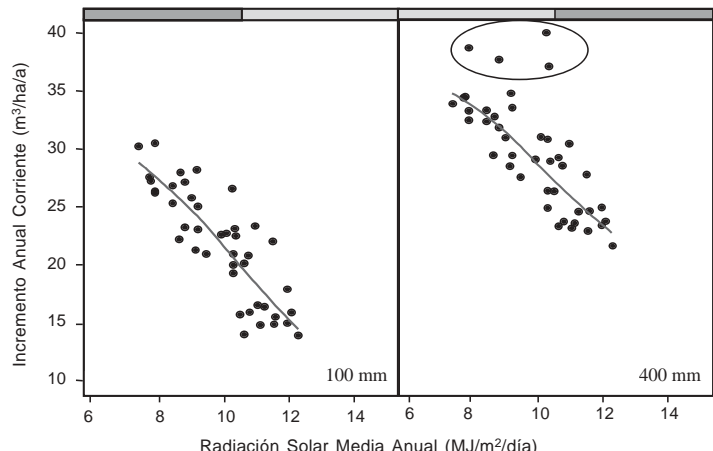

Figura 7. Relación entre incremento anual corriente en volumen y la radiación solar, para suelos con 100 y $400 \mathrm{~mm}$ de capacidad de almacenamiento de agua. Relationship between current annual increment in volume and solar radiation. Soils with 100 and $400 \mathrm{~mm}$ of available water storage capacity.

en septiembre (figura 9). En el período septiembre-diciembre, existen muchas zonas que aún no experimentan un estrés hídrico severo (figuras 5 y 6); sin embargo, variaciones en crecimiento en este período aparentemente están relacionadas con el déficit de presión de vapor y temperatura (figuras 5 y 6 ).

Finalmente, las figuras 10 y 11 muestran la distribución espacial de la productividad de pino radiata estimada con 3-PG para suelos con 100 y $400 \mathrm{~mm}$ de capacidad de almacenamiento de agua. La parte alta de la cordillera de Nahuelbuta, precordillera y cordillera de Los Andes no pre-

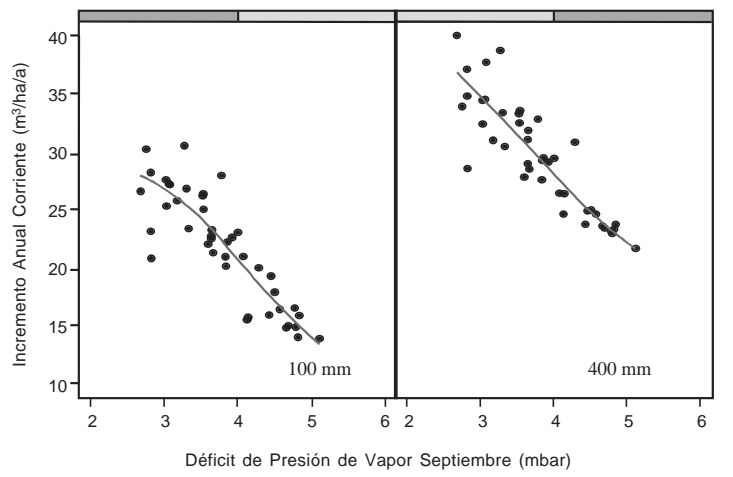

Figura 9. Relación entre incremento anual corriente en volumen y déficit de presión de vapor, para suelos con 100 y $400 \mathrm{~mm}$ de capacidad de almacenamiento de agua.

Relationship between current annual increment in volume and vapor pressure deficit. Soils with 100 and $400 \mathrm{~mm}$ of available water storage capacity.

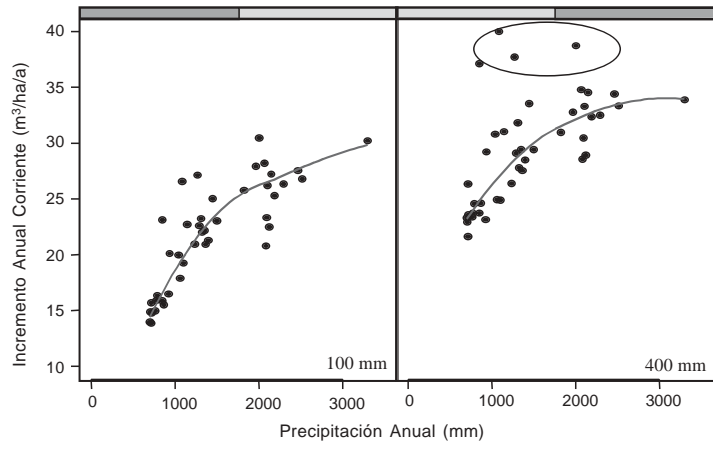

Figura 8. Relación entre incremento anual corriente en volumen y precipitación anual, para suelos con 100 y $400 \mathrm{~mm}$ de capacidad de almacenamiento de agua.

Relationship between current annual increment in volume and annual precipitation. Soils with 100 and $400 \mathrm{~mm}$ of available water storage capacity.

sentan valores por estar fuera del rango de distribución de pino radiata. En la figura 11 se observan claramente las zonas de mayor eficiencia productiva ubicadas en la zona costera. Sin embargo, en los suelos con baja capacidad de almacenamiento de agua estas diferencias no son tan claras (figura 10), puesto que el período seco es mayor y predomina por sobre otros factores como el déficit de presión de vapor.

Efecto de la capacidad de almacenamiento de agua disponible del suelo sobre la productividad: El efecto de la capacidad de almacenamiento de agua disponible del suelo sobre el incremento anual corriente en volumen de pino radiata es considerable. La figura 12, muestra un claro aumento en la productividad a medida que aumenta la capacidad de almacenamiento de agua del suelo. Esto muestra que la productividad potencial dentro de cada zona climática varía de acuerdo a la capacidad de almacenamiento de agua del suelo, esta última es función de la textura, porcentaje de fragmentos rocosos y profundidad del suelo, principalmente.

Por otra parte, el rango de valores dentro de cada clase de capacidad de almacenamiento de agua es siempre amplio, y aumenta desde $16 \mathrm{~m}^{3} \mathrm{ha}^{-1}$ año ${ }^{-1}$ a los $50 \mathrm{~mm}$, hasta $20 \mathrm{~m}^{3} \mathrm{ha}^{-1} \mathrm{año}^{-1}$ a los $500 \mathrm{~mm}$. Esto indica que las variaciones climáticas a lo largo del rango de distribución de pino radiata en Chile ejercen un fuerte control sobre la productividad de las plantaciones. Además, existen sitios con un mayor aumento de la productividad 


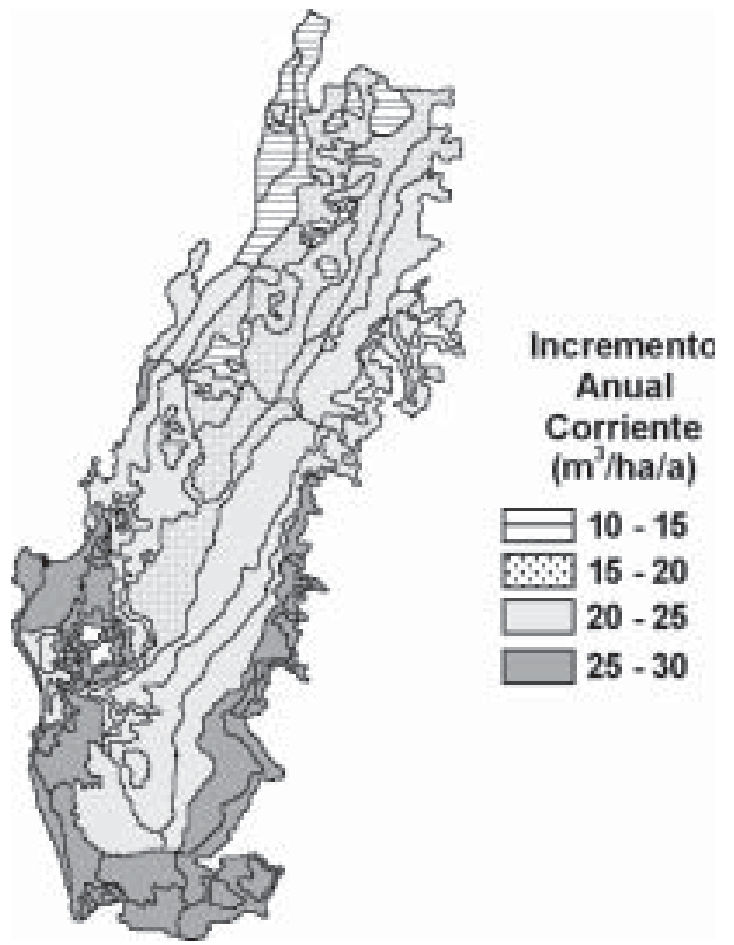

Figura 10. Mapa de productividad de pino radiata en suelos con $100 \mathrm{~mm}$ de capacidad de almacenamiento de agua.

Map of radiata pine productivity for soils with $100 \mathrm{~mm}$ of available water storage capacity.

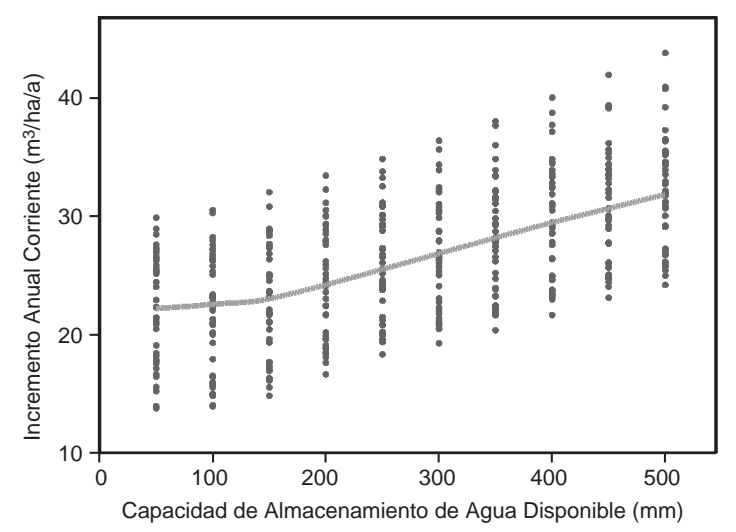

Figura 12. Efecto de la capacidad de almacenamiento de agua del suelo en el incremento anual corriente en volumen de pino radiata.

Soil-water storage capacity effect on current annual increment in volume of radiata pine.

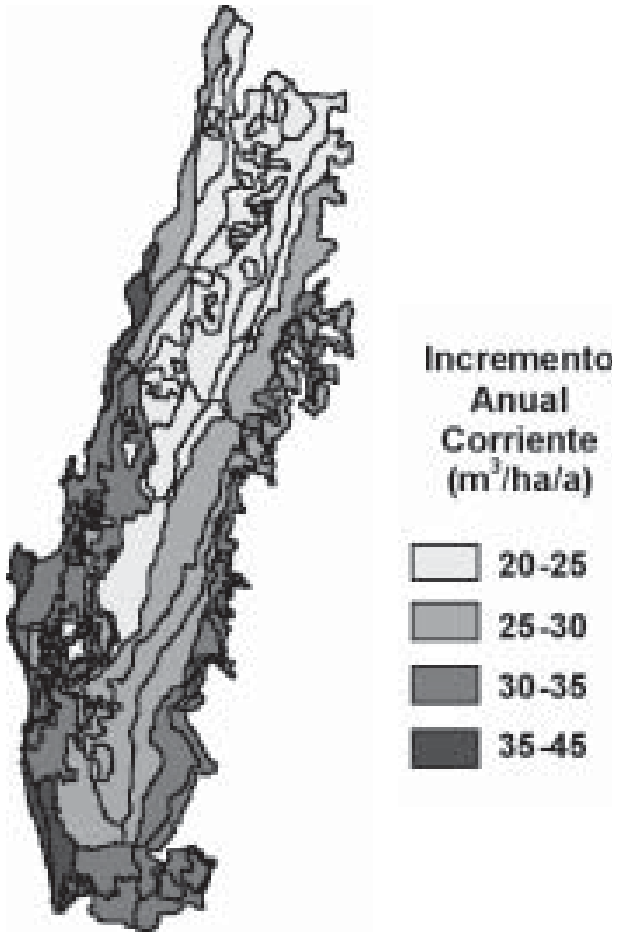

Figura 11. Mapa de productividad de pino radiata en suelos con $400 \mathrm{~mm}$ de capacidad de almacenamiento de agua.

Map of radiata pine productivity for soils with $400 \mathrm{~mm}$ of available water storage capacity.

a medida que aumenta la capacidad de almacenamiento de agua.

La capacidad de almacenamiento de agua del suelo determina la cantidad de agua, producto de las precipitaciones, que puede ser almacenada y utilizada por los árboles. Esto es muy importante en climas mediterráneos, en los cuales las precipitaciones se concentran en invierno, cuando la evapotranspiración es baja, y en donde el período seco ocurre en verano, cuando la evapotranspiración es alta. De este modo, mientras más agua pueda almacenar el suelo durante el invierno, más tarde se produce el estrés hídrico que provoca el cierre de los estomas y reduce la eficiencia fotosintética. Esto puede ser claramente observado en la figura 13, que muestra el valor mensual del modificador de agua del suelo de 3-PG, para el distrito agroclimático 7.1, ubicado en la costa al sur de Constitución. A medida que disminuye este modificador, disminuye la conductancia del dosel (respecto del máximo posible) y con ello se 
reduce la eficiencia fotosintética. En un suelo capaz de almacenar sólo $100 \mathrm{~mm}$ de agua disponible, el estrés hídrico reduce dramáticamente las tasas de fotosíntesis a partir de diciembre, comenzando a recuperarse sólo en mayo del año siguiente. En el caso de un suelo más profundo, capaz de almacenar $400 \mathrm{~mm}$ de agua disponible, el estrés hídrico es severo sólo a partir de febrero y el mínimo nunca es tan bajo como en el caso anterior. Esto indica que, en el caso del suelo profundo, gran parte de la radiación solar interceptada durante diciembre y enero puede ser utilizada para producir materia seca, a diferencia de lo que ocurre con el suelo delgado, cuya capacidad de almacenar agua disponible es de sólo $100 \mathrm{~mm}$.

De acuerdo al modelo, los factores de suelo y sitio que determinan el desarrollo del estrés hídrico (modificador de agua del suelo) incluyen la precipitación, la cantidad de agua que puede almacenar el suelo, la radiación solar y de la demanda evaporativa del aire. Si el suelo puede almacenar poca agua, es posible que las plantaciones estén bajo estrés hídrico incluso si la precipitación anual es alta. En suelos con baja capacidad de almacenamiento de agua disponible, el estrés hídrico a partir de diciembre puede ser significativo $(70 \%$ de la máxima eficiencia en el uso de la luz), incluso si la precipitación anual es superior a los $1.000 \mathrm{~mm}$ (figura 14). Esto se debe a la estacio-

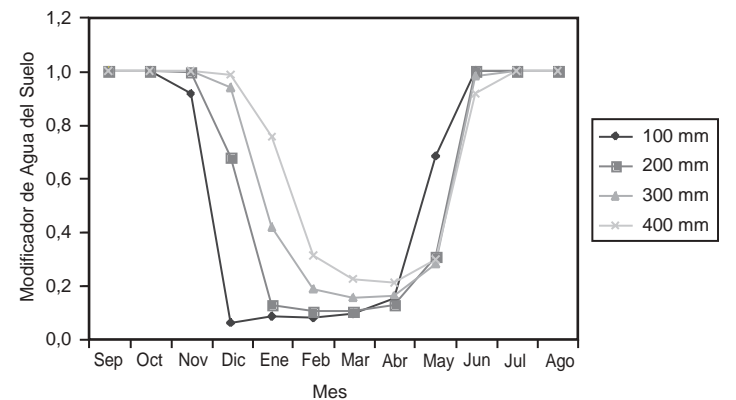

Figura 13. Variación mensual del modificador de agua del suelo de 3-PG para suelos con distinta capacidad de almacenamiento de agua, en la zona 7.1, ubicada en la costa al sur de Constitución.

Monthly variation in 3-PG soil water modifier for soils with different water storage capacity in area 7.1, located on the coast south of Constitución. nalidad de las precipitaciones, que se concentran en invierno.

El efecto de la capacidad de almacenamiento de agua sobre la productividad de pino radiata cambia a lo largo del rango de distribución de la especie en Chile. En zonas con baja precipitación, la capacidad de almacenamiento de agua del suelo tiene un impacto mayor en la productividad de pino radiata que en zonas de alta precipitación. La figura 15 muestra el porcentaje de incremento de la productividad asociado a un aumento de la capacidad de almacenamiento de agua del suelo desde 100 a $400 \mathrm{~mm}$. Como se observa en esta figura, en zonas de baja precipitación es posible tener incrementos en productividad cercanas al $60 \%$ asociadas al aumento de $300 \mathrm{~mm}$ en la capacidad de almacenamiento de agua del suelo. El efecto de la capacidad de almacenamiento de agua disminuye en forma prácticamente lineal a medida que aumenta la precipitación anual, desde $600 \mathrm{~mm}$ hasta aproximadamente $1.500 \mathrm{~mm}$.

Estos resultados concuerdan con las evidencias empíricas presentadas por Gerding y Schlatter (8). Las diferencias en productividad dentro de cada zona climática que ellos presentan varían desde $28 \%$, en climas de la costa occidental con influencia mediterránea (alta precipitación) hasta $81 \%$, en climas templado cálidos con estación seca prolongada (baja precipitación). Por tratarse de

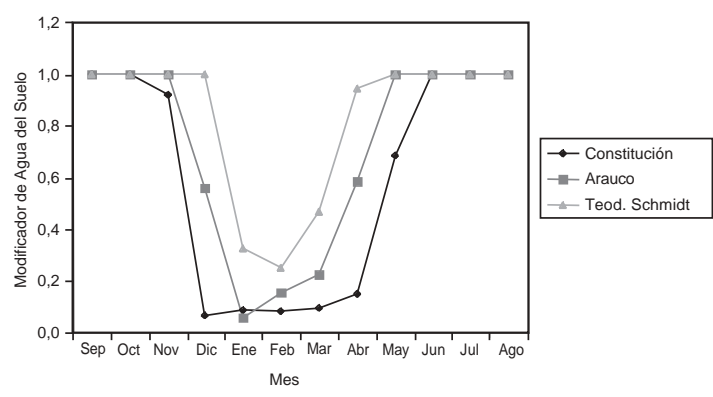

Figura 14. Variación mensual del modificador de agua del suelo de 3-PG para suelos con $100 \mathrm{~mm}$ de capacidad de almacenamiento de agua disponible, en tres zonas con distinta precipitación (valores en paréntesis): la costa de Constitución (840 mm), Arauco (1.260 $\mathrm{mm}$ ) y alrededor de la localidad de Teodoro Schmidt $(1.990 \mathrm{~mm})$

Monthly variation in 3-PG soil water modifier for soils with $100 \mathrm{~mm}$ of available water storage capacity in three areas with contrasting precipitation (values in parentheses) on the coast at Constitución $(840 \mathrm{~mm})$, Arauco $(1260 \mathrm{~mm})$, and Teodoro Schmidt (1990 mm). 


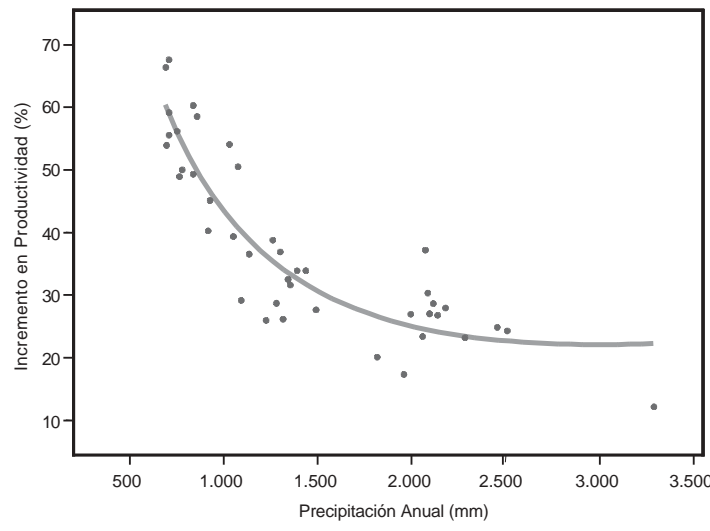

Figura 15. Porcentaje de incremento de la productividad asociado a un aumento de la capacidad de almacenamiento de agua del suelo desde 100 a $400 \mathrm{~mm}$.

Percentage increase in productivity with an increase in soil water storage capacity from 100 to $400 \mathrm{~mm}$.

valores empíricos, los valores presentados por Gerding y Schlatter (8) incluyen no sólo el efecto del clima, sino también diferencias en fertilidad y manejo de las plantaciones, sin embargo, muestran una tendencia y magnitud similar a las observadas en la figura 15.

El mapa en la figura 16 muestra la distribución espacial del porcentaje de incremento en la productividad de pino radiata presentado en la figura 15. Se puede apreciar claramente que en todas las zonas con baja precipitación, menor a $1.000 \mathrm{~mm}$, ubicadas en la parte norte del área de distribución hasta aproximadamente el límite entre las Regiones VII y VIII, el porcentaje de incremento en la productividad es superior al $40 \%$. Esta zona de alto incremento en la productividad se extiende en la VIII Región, a lo largo del Valle Central y vertiente oriental de la Cordillera de la Costa.

Implicancias para el manejo de plantaciones: Al considerar todas las zonas climáticas, el modificador de agua del suelo es el que presenta los valores más bajos, indicando que el estrés hídrico es probablemente el factor más importante que controla la productividad de pino radiata en Chile. Esto concuerda con los resultados experimentales en otras zonas con plantaciones de pino radiata en clima mediterráneo $(27,28,29)$, que muestran cómo el control de malezas reduce significativamente el estrés hídrico al que están sometidas las plantas y produce grandes respuestas en crecimiento. En Chile, los resultados de Kogan

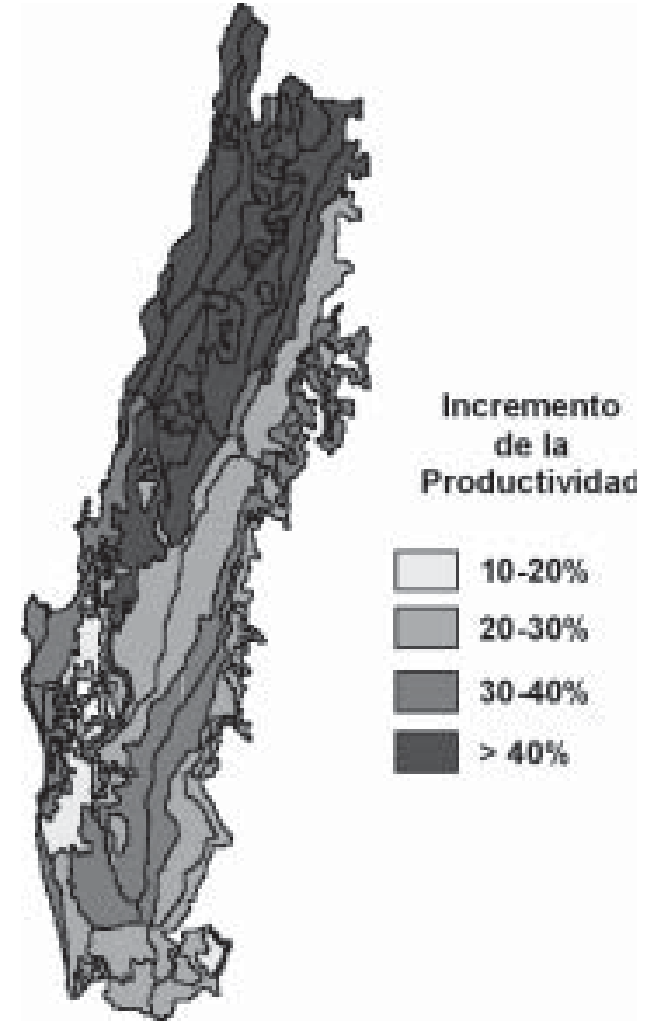

Figura 16. Distribución espacial del incremento de la productividad asociado a un aumento de la capacidad de almacenamiento de agua del suelo desde 100 a $400 \mathrm{~mm}$.

Spatial distribution of the increase in productivity associated with an increase in soil water storage capacity from $100 \mathrm{~mm}$ to $400 \mathrm{~mm}$.

et al. (10) en el área de Constitución muestran una gran respuesta al control de malezas, lo que concuerda con lo encontrado en zonas con condiciones climáticas similares. De acuerdo al modelo 3PG (14), los factores del sitio que determinan la magnitud y duración del período de estrés hídrico incluyen precipitación, capacidad del suelo para almacenar agua disponible, radiación solar y demanda evaporativa del aire. Si bien estos factores no pueden ser modificados a través del manejo, información acerca de la variación espacial y temporal de éstos, puede ser muy valiosa para establecer los niveles superiores de productividad en plantaciones forestales. Debido a esto, parece fundamental contar con información meteorológica con una resolución espacial superior a la utilizada en este estudio, donde se usaron grandes distritos agroclimáticos. Dada la importancia del clima en 
el crecimiento de pino radiata, es recomendable contar con información meteorológica con mayor resolución espacial, que permita generar mapas continuos de cada una de las variables importantes para el crecimiento de los bosques (en reemplazo de unidades agroclimáticas discretas).

Una vez determinados los niveles de productividad potencial, es posible comparar esos valores con los valores de productividad actual y de esta manera tener una estimación de la magnitud de las ganancias que se pueden realizar a través del manejo (30). El amplio rango de productividad estimada a través de 3-PG concuerda con las evidencias empíricas presentadas por Gerding y Schlatter (8). Ellos muestran grandes diferencias en productividad incluso dentro de una misma zona climática o tipo de suelo. Esto sugiere que existen oportunidades para incrementar la productividad actual de pino radiata en gran parte del rango de distribución de la especie en Chile.

Desde el punto de vista del manejo, la magnitud y duración del período de estrés hídrico pueden ser modificadas a través de podas, raleos, preparación de suelo y control de malezas (27, 28). Adicionalmente, los resultados de este estudio sugieren que el riego, de ser posible, debe ser considerado como una opción. Como la disponibilidad de agua juega un rol tan importante en el crecimiento de pino radiata en Chile, es muy probable que exista una interacción entre los tratamientos silvícolas que afectan la disponibilidad de agua y aquellos que afectan la disponibilidad de nutrientes. Estas interacciones van a ser muy importantes al interpretar, por ejemplo, la magnitud y duración de la respuesta a tratamientos de fertilización. Claramente, para lograr optimizar la productividad de pino radiata en Chile, es necesario entender mejor los factores que determinan el estrés hídrico y su interacción con los regímenes de manejo.

\section{AGRADECIMIENTOS}

Nuestros agradecimientos a José Alvarez (Forestal Mininco) y Cristian Montes (Bioforest S.A.) por los valiosos comentarios durante el desarrollo de este proyecto. Agradecemos también a R. Waring y J. Landsberg por facilitarnos el modelo, y además a este último por sus valiosas sugerencias y comentarios en el uso y parametrización del modelo para pino radiata.

\section{BIBLIOGRAFIA}

(1) JARVIS, P. G., J. W. LEVERENZ. Productivity of temperate, deciduous and evergreen forests. In: LANGE, O. L., P. S. NOBEL, C. B. OSMOND and H. ZIEGLER Physiological Plant Ecology IV. Encyclopedia of plant physiology. New York: Springer-Verlag, 1983, vol. 12D, p. 233-280.

(2) LINDER, S. Potential and actual production in Australian forest stands. In: LANDSBERG, J. J. AND PARSONS, W. Research for Forest Management. Melbourne: CSIRO, 1985, p. 11-35.

(3) ALBAUGH, T. J., H. L. ALLEN, P. M. DOUGHERTY, L. W. KRESS, J. S. KING. Leaf area and above- and belowground growth responses of loblolly pine to nutrient and water additions. Forest Science, 1998, vol. 44, p. 317-328.

(4) ALLEN, H. L AND T. J. ALBAUGH. Ecophysiological basis for plantation production: a loblolly pine case study. Bosque (Chile), 1999, vol. 20, $\mathrm{N}^{\circ}$ 1, p. 3-8.

(5) LANDSBERG, J. J. The use of models in interpreting plant response to weather. In: J. GRACE, E. D. FORD and P. G. JARVIS. Plants and their atmospheric environment. Oxford: Blackwell Scientific Publications, 1981, p. 369-389.

(6) MAKELA, A, J. J. LANDSBERG, A. R. EK, T. E. BURK, M. TER-MIKAELIAN, G. I. AGREN, C. D. OLIVER, P. PUTTONEN. Process-based models for forest ecosystem management: current state of the art and challenges for practical implementation. Tree Physiology, 2000, vol. 20, p. 289-298.

(7) LANDSBERG, J. J., R. H. WARING, N. C. COOPS. Performance of the forest productivity model 3-PG applied to a wide range of forest types. Forest Ecology and Management, 2003, vol. 172, p. 199-214.

(8) GERDING, V., J. E. SCHLATTER. Variables y factores del sitio de importancia para la productividad de Pinus radiata D. Don en Chile. Bosque (Chile), 1995, vol. $16, \mathrm{~N}^{\mathrm{o}} 2$, p. 39-56.

(9) KOGAN, M., R. FIGUEROA. Interferencia producida por las malezas durante los dos primeros años Pinus radiata D. Don. Bosque (Chile), 1999, vol. 20, No 1 p. 57-64.

(10) KOGAN, M., R. FIGUEROA, H GILABERT. Weed control intensity effects on young radiata pine growth Crop Protection, 2002, vol. 21, p. 253-257.

(11) ALVAREZ, J., J. RODRIGUEZ, D. SUAREZ. Mejoramiento de la productividad de plantaciones de Pinus radiata $\mathrm{D}$. Don, a través de un método racional de fertilización. Bosque (Chile), 1999, vol. 20, No 1, p. 23-36.

(12) ALLEN, H. L. Silvicultural Treatments to Enhance Productivity. In: J. EVANS. The Forests Handbook. Oxford: Blackwell Science Ltd., 2001, vol. 2, p. 129 139.

(13) SANTIBAÑEZ, F., J. URIBE. Atlas agroclimático de Chile. VI, VII, VIII y IX Regiones. Santiago: Laboratorio de Agroclimatología, Facultad de Ciencias Agrarias y Forestales, Universidad de Chile, 1993, 73 p.

(14) LANDSBERG, J. J., R. H. WARING, A generalised model of forest productivity using simplified concepts of radiation-use efficiency, carbon balance and partitioning. Forest Ecology and Management, 1997, vol. 95, p. 209-228

(15) COOPS, N. C. Improvement in predicting stand growth of Pinus radiata (D. Don) across landscapes using NOAA AVHRR and Landsat MSS imagery combined with a 
BOSQUE 25(3): 11-24, 2004

Efectos del clima y capacidad de almacenamiento de agua del suelo en productividad de rodales de pino radiata en Chile...

forest growth process model (3-PGS). Photogrammetric Engineering and Remote Sensing, 1999, vol. 65, p. 11491156.

(16) WARING, R. H. A process model analysis of environmental limitations on the growth of Sitka spruce plantations in Great Britain. Forestry, 2000, vol. 73 $\mathrm{N}^{\circ} 1$, p. $65-78$.

(17) COOPS, N. C., R. H. WARING. Estimating forest productivity in the eastern Siskiyou Mountains of southwestern Oregon using a satellite driven model, 3-PGS. Canadian Journal of Forest Research, 2001, vol. 31, p. 143-154.

(18) LANDSBERG, J. J, K. H. JOHNSON, T. J. ALBAUGH, H. L. ALLEN, S. E. MCKEAND. Applying 3-PG, a simple process-based model designed to produce practical results, to data from loblolly pine experiments. Forest Science, 2001, vol. 47, p. 43 - 51

(19) SANDS, P. J., J. J. LANDSBERG. Parameterisation of 3-PG for plantation grown Eucalyptus globulus. Forest Ecology and Management, 2002, vol. 163, p. 273-292.

(20) TICKLE, P. K., N. C. COOPS, S. D. HAFNER, THE BAGO SCIENCE TEAM. Assessing forest productivity at local scales across a native eucalypt forest using a process model, 3PG-SPATIAL. Forest Ecology and Management, 2001, vol. 152, p. 275-291.

(21) WARING, R. H. J. J LANDSBERG, M WILLIAMS. Net primary production of forests: a constant fraction of gross primary production? Tree Physiology, 1998, vol 18 , p. $129-134$

(22) WHITEHEAD, D., F. M. KELLIHER, C. M FRAMPTON, M. J. S. GODFREY. Seasonal development of leaf area in a young, widely spaced Pinus radiata
D. Don stand. Tree Physiology, 1994, vol. 14, p. 1019 1038 .

(23) JARVIS, P. G. Stomatal conductance, gaseous exchange and transpiration. In: J. GRACE, E.D. FORD AND P.G. JARVIS. Plants and their atmospheric environment. Oxford: Blackwell Scientific Publications, 1981, p. 175-204.

(24) PETERS, R, M. JOBET, A. AGUIRRE Compendio de tablas auxiliares para el manejo de plantaciones de pino insigne. Manual $\mathrm{N}^{\circ} 14$. Santiago: INFOR, 1985, $140 \mathrm{pp}$.

(25) NAMBIAR, E. K. S. Interplay between nutrients, water, root growth and productivity in young plantations. Forest Ecology and Management, 1990, vol. 30, p. 213-232.

(26) BEETS, P. N., K. A. ROBERTSON, J. B. FORDROBERTSON, J. GORDON, J. P. MACLAREN. Description and validation of C_CHANGE: a model for simulating carbon content in managed Pinus radiata stands. New Zealand Journal of Forestry Science, 1999 vol. 29, $\mathrm{N}^{\circ} 3$, p. 409- 427.

(27) NAMBIAR, E. K. S., P. G. ZED. Influence of weeds on the water potential, nutrient content and growth of young radiata pine. Australian Forest Research, 1980, vol. 10, p. $279-288$

(28) NAMBIAR, E. K. S. Management of forest under nutrient and water stress. Water, Air and Soil Pollution, 1990/ 91 , vol. 54 , p. $209-230$.

(29) NAMBIAR, E. K. S. Productivity and sustainability of plantation forests. Bosque (Chile), 1999, vol. 20, No 1 p. 9-21.

(30) SAMPSON, D. A., H. L. ALLEN. Regional influences of soil available water and climate, and leaf area index on simulated loblolly pine productivity. Forest Ecology and Management, 1999, vol. 124, p. 1-12. 